\title{
Screening pulmonary arteriovenous malformations in a large cohort of Spanish patients with hemorrhagic hereditary telangiectasia.
}

José Antonio Parra. MD, PhD. Department of Radiology Hospital Universitario Marqués de Valdecilla. Santander, Cantabria, Spain. Associate professor. University of Cantabria. japarra@humv.es

José María Cuesta. MD. Department of Cardiology. Hospital Sierrallana. Torrelavega, Cantabria, Spain. jmccbuelna@hotmail.com

Roberto Zarrabeitia. MD, PhD. Department of Internal Medicine. Hospital Sierrallana and Centro de Investigación Biomédica en Red de Enfermedades Raras (CIBERER). Torrelavega, Cantabria, Spain. roberto.zarrabeitia@scsalud.es

Concepción Fariñas-Álvarez MD, PhD. Quality Unit. Hospital Universitario Marques de Valdecilla. Santander, Cantabria, Spain.cfarinas@humv.es

Jesús Bueno. MD, PhD. Department of Radiology. Hospital Sierrallana. Torrelavega, Cantabria, Spain.jesus.bueno@scsalud.es

Sarah Marqués. MD. Department of Radiology. Hospital Sierrallana. Torrelavega, Cantabria, Spain.sarah.marques@scsalud.es

Carmen Parra-Fariñas. MD. Department of Radiology. Hospital Universitari Vall d’Hebron. Barcelona. Catalonia, Spain.carmenparrafarinas@gmail.com María Luisa Botella. PhD. Centro de Investigaciones Biológicas, Consejo Superior de InvestigacionesCientíficas (CSIC), and Centro de InvestigaciónBiomédica en Red de EnfermedadesRaras (CIBERER), Madrid, Spain. cibluisa@cib.csic.es.

Carmelo Bernabéu. PhD. Centro de InvestigacionesBiológicas, Consejo Superior de InvestigacionesCientíficas (CSIC), and Centro de InvestigaciónBiomédica en Red de EnfermedadesRaras (CIBERER). Madrid, Spain. CIBERER. bernabeu.c@cib.csic.es 
Jesús Zarauza. MD, PhD. Department of Cardiology. Hospital UniversitarioMarqués de Valdecilla. Santander, Cantabria, Spain. manuel.zarauza@humv.es

All authors takes responsibility for all aspects of the reliability and freedom from bias of the data presented and their discussed interpretation".

\section{Corresponding author:}

Dr José Antonio Parra

Department of Radiology

Hospital Universitario Marqués de Valdecilla

Avd. Valdecilla s/n. Santander, 39008. Cantabria. Spain

e-mail:japarra@humv.es

Phone 0034942202520

This study has been supported by grants from Instituto de Salud Carlos III (ISCIII; PI11/0246 to JAP), FEDER (to JAP), Ministerio de Economía y Competitividad of Spain (SAF2011-23475 to LMB and SAF2013-43421-R to CB), and Centro de Investigación Biomédica en Red de Enfermedades Raras (CIBERER; ISCIIICB06/07/0038 to CB). CIBERER is an initiative of the Instituto de Salud Carlos III (ISCIII) of Spain supported by FEDER funds.

All authors declare no conflict of interest 


\section{Key words}

Hereditary hemorrhagic telangiectasia

Pulmonary arteriovenous malformations

Transthoracic contrast echocardiography

Computed tomography

Ultrasound contrast agents 


\section{ABSTRACT}

\section{Background and objectives}

Because of serious nature of potential complications, screening for pulmonary arteriovenous malformations is required in patients with hereditary hemorrhagic telangiectasia. The aim of this study was to evaluate the utility of contrast echocardiography and compare the performance of two contrast agents: agitated saline and Gelofusine.

\section{Material and Methods}

Two hundred and five patients screened for PAVMs using TTCE and computed tomography (CT) performed with an interval of less than 180 days. Contrast echocardiography studies were graded on a 4-point semiquantitative scale based on the amount of microbubbbles seen in left heart chambers.

\section{Results}

Positive TTCE findings were seen in 137 (66.8\%) patients, whereas CT confirmed PAVMs in 59 (43.1\%). Two of 67 grade 1 patients; 18 of 42 grade 2; 17 of 22 grade 3 and all grade 4 had PAVMs on CT. Embolotherapy was feasible in 38.9\% patients in grade 2 and $82.3 \%$ and $95.2 \%$ in grade $3-4$. No patients in grade 1 were embolized. The mean cardiac cycle in which bubbles were first seen in the left heart in patients without and with PAVMs on CT was 6.1 and $3.9(P<0.0001)$. Compared to saline, Gelofusine produced an overall increase in grade.

\section{Conclusions}

No grade 1 patients had treatable PAVMs. There is a need for improvement in the selection of patients for CT in grade 2, where less than half have PAVMs on CT. The cardiac cycle may help to differentiate between patients with and without PAVMs. Gelofusine was not better than saline for PAVM screening. 


\section{Introduction}

Hereditary hemorrhagic telangiectasia (HHT) is a rare (1 in 5000 to 8000 people) disorder, transmitted in an autosomal dominant trait; with more than $90 \%$ of the cases caused by mutations in Endoglin (ENG; HHT1), activin A receptor type II-like 1 (ACVRL1/ALK1; HHT2), or SMAD4 ( Hereditary hemorrhagic telangiectasia and Juvenile polyposis; HTJP) genes $(1,2)$. These mutations, together with other environmental factors promote the development of small (telangiectasia) or large arteriovenous malformations (AVMs) in almost all organs of the economy $(1,2)$; including the lung, where pulmonary arteriovenous malformations (PAVMs) are a common finding. This is especially true among HHT1 patients in whom pulmonary shunts are seen in up to $85 \%$ of these patients (3-7). PAVMs create a right-to-left shunt between the pulmonary arterial and venous circulation that can lead to hypoxemia, paradoxical embolism, or rupture and, as a consequence, not infrequently a brain abscess, stroke, or hemoptysis are seen in individuals with a previously undiagnosed PAVM (2-4). Recent data also, highlight that reduced oxygenation of blood due to pulmonary AVMs results in a graded erythrocytotic response to maintain arterial oxygen content, and higher stroke volumes and/or heart rates to maintain oxygen delivery (8). This latter situation can also be aggravated in HHT patients by the anemia resulting from iron losses due to bleeding from nasal and gastrointestinal telangiectasies. (8). Anatomic resolution of PAVMs through embolization, has been shown to reduce strokes, migraines, and hematologic, cardiac and ventilatory demands (2).

Because of the serious nature of these potential complications and benefits for embolization screening for PAVMs is recommended in HHT patients (9). 
Transthoracic contrast echocardiography (TTCE) followed by CT in patients testing positive with this technique are the recommended imaging methods in the last 2010 clinical guideline (9). Nonetheless, positive TTCE findings are relatively common in patients with HHT $(60 \%$ to $85 \%$ of patients tested) $(3,10,11)$, whereas a much smaller percentage of between $21 \%$ and $52.3 \%$ actually show PAVMs on CT (12). Therefore, adherence to clinical guideline recommendations would result in a large number of unwarranted CT studies, implying unnecessary radiation exposure and healthcare expenditure (2,13-15). The results of a previous study by our group (13) and those of other authors $(10,14,16)$ have indicated that use of a system to grade the passage of contrast material on TTCE to estimate the size of pulmonary shunts is of value for selecting patients who should subsequently undergo CT evaluation. Two TTCE grading systems have been proposed: a 4-grade scale $(10,13,17)$ and a 3 -grade scale $(7,14,16)$. The main difference between the two options is that grades 3 and 4 in the 4 -grade scale correspond to grade 3 in the 3-grade scale. At present, the indication for CT seems clear in patients showing moderate or extensive contrast passage using these scales (grades 2 , 3, and 4). In patients with a pulmonary shunt grade 1, according to the work of Velthuis et al (18), a conservative management strategy (without CT and antibiotic prophylaxis) is probably justified considering the absence of treatable PAVMs and the negligible risk of cerebral paradoxical embolism in this subset of patients. An interesting question in TTCE grade 1 is to know if these positive results are a consequence of very small PAVMs or the results of normally present intrapulmonary arteriovenous anastomosis (19). An increase in cardiac output has been shown to produce an increase of blood flow through to this normally present intrapulmonary arteriovenous anastomosis in healthy patients (20). 
Most echocardiography studies carried out in HHT patients use an agitated saline solution as the contrast agent. However, other contrast options are available, such as the colloid solution, Gelofusine (Braun Medical, Melsungen, Germany). Gelofusine is reported to provide better opacification of the heart chambers than agitated saline solution (21), as the bubbles formed are smaller $(22,23)$ and more stable (24). Use of Gelofusine may result in a smaller number of suboptimal studies relative to saline contrast $(21,24)$.

Based on this background, the aim of this study was to assess the use of graded contrast echocardiography for selecting Spanish HHT patients to undergo CT, and evaluate the performance of agitated saline solution versus Gelofusine as contrast media. To the best of our knowledge, this is the first study comparing these two contrast agents in the screening of PAVMs of HHT patients.

\section{Materials and Methods}

From January 2003 to December 2014, 664 patients with suspected HHT were seen in our HHT unit through one of the following channels: ambulatory visit, hospital admittance, or genetic testing alone. Patients seen through either of the first 2 channels underwent a hospital protocol that included a clinical historytaking, physical examination, laboratory testing, genetic testing, TTCE, chest or abdominal CT, abdominal ultrasound, and brain or lumbar MRI, when clinically justified. Only patients who underwent TTCE and CT within an interval of $\leq 180$ days between the two techniques were included in the study. Patients in whom a persistent foramen ovale was detected were excluded. The hospital ethics committee for clinical research of the Autonomous Community of Cantabria 
approved the study protocol. All patients provided written consent for participation. The presence or absence of anemia was considered in women and men when hemoglobin (Hb) levels were lower than $12 \mathrm{~g} / \mathrm{dL}$, and 13,5 g/dL, respectively

The TTCE technique, previously used by our team and described by others (13, 14), was performed by 3 experienced echocardiographers, following intravenous line placement in an antecubital vein. The two contrast agents, agitated saline ( 9 $\mathrm{ml}$ saline solution mixed with $0.5 \mathrm{ml}$ room air and $0.5 \mathrm{ml}$ of blood from the patient) and Gelofusine $(10 \mathrm{ml})$, were administered with the patient in left lateral decubitus. A four-chamber view was used, and TTCE was considered positive for pulmonary shunting when bubbles were observed in the left atrium without application of the Valsalva maneuver. The number of cardiac cycles that occurred before bubbles appeared in the left atrium was recorded and a delay of $>3$ cycles was established to differentiate PAVMs from intracardiac shunt (25). Shunts visualized through a pulmonary vein were classified as pulmonary shunts regardless of the cardiac cycle (12). A second study with Valsalva manoeuvre was performed when contrast was present in the left atrium in less than four cardiac cycles. The study was considered positive for PAVM if intracardiac shunts were not visualized by color Doppler and the pattern of appearance of bubbles was not modified by Valsalva. Based on the amount of bubbles visible in the left ventricle, TTCE findings were rated according to the grading system of Barzilai et al (17), modified by Zukotynski et al. (10) and used in our previous study (13) into x4 grades: grade 1, minimal left ventricular opacity (up to 20 bubbles); Grade 2, 
moderate opacity; grade 3, extensive opacity without outlining the endocardium; and grade 4, extensive opacity with endocardial definition.

TTCE with agitated saline contrast was performed in all patients. Between 2008 and 2014, patients additionally underwent TTCE using Gelofusine to determine whether there were differences in screening outcomes versus saline. The same protocol described above was used, and the Gelofusine study was performed a few minutes after the saline study in the same session after making sure that there were no bubbles from the previous study.

Chest CT was carried out on 3 different multidetector systems over the study period: a 2-row Somaton AR.T scanner (Siemens, Erlangen, Germany), a 32-row LightSpeed scanner (General Electric, New York, USA), and an 80-row Aquilion Prime TSX-303A scanner (Toshiba Medical Systems Corporation, Japan), using $\leq 3$ mm slice thickness (9). Nonionic contrast agent $(300 \mathrm{mg} / \mathrm{mL}$ iodine at a rate of 3 $\mathrm{mL} / \mathrm{sec}$ and maximum dose of $2 \mathrm{~mL} / \mathrm{kg}$ ) was routinely administered because in most of these patients the liver was included in the study in order to rule out hepatic arteriovenous malformations (HAVMs). These studies were performed in a caudo-craneal direction and always included an image acquisition in an early arterial phase $(5,26)$. The images obtained were evaluated by 3 radiologists with experience in HHT. Visualization of a nodule with an afferent artery and efferent vein was considered diagnostic for PAVM (5). The presence of telangiectases, perfusion disorders, vascular confluent masses or arteriovenous or veno-venous shunts were considered diagnostic for HAVMs (27). In the assessments of TTCE and CT features, readers were blinded to the results of the other imaging study. Revascularization of a previously treated PAVM was defined based on CT findings of a persistent aneurysmal sac with prominent afferent arteries and drainage veins 
$(28,29)$. Patients with an afferent artery measuring $\geq 3 \mathrm{~mm}$, a PAVM with less size but suitable for embolization and those with CT criteria indicating revascularization (30) were sent to the angiography suite to plan embolotherapy.

\section{Statistical analysis}

Baseline characteristics of the patients were compared using a Mann-Whitney U test or Kruskal-Wallis test for continuous variables. For categorical variables the chi-square test or Fisher exact test when expected values in any of the cells of a contingency table were below 5 were calculated (Fisher exact Test were estimated in case of $m \times n$ tables by Monte Carlo method). Kappa statistics with $95 \%$ confidence intervals (95\% CI) were used to assess agreement between TTCE studies performed using agitated saline or Gelofusine solution. Sensitivity, specificity, and positive and negative predictive values were calculated, using the CT results as the reference test; 95\% CI were calculated as exact binomial confidence intervals. The diagnostic accuracy of cardiac cycle was evaluated by constructing a receiver-operating-characteristic (ROC) curve. $P$-values $<0.05$ (twosided) were considered statistically significant. Statistical analyses were performed using SPSS v 19.0 (IBM Corp, Armonk, NY), and Stata statistical software (Release 10.0, Stata Corporation, College Station, TX).

\section{Results}

Of the 664 patients evaluated in the HHT Unit of Hospital Sierrallana (Cantabria, Spain), 449 (65.2\%) were diagnosed with HHT based on clinical criteria or genetic testing. Among this total, 312 (69.5\%) patients had TTCE to rule out PAVMs, and $205(45.7 \%)$ underwent both TTCE and chest CT at a mean interval between 
assessments of 14 days (SD 34.8, range 0-180 days). There were no significant differences in demographic and genetic characteristics between the overall population and those undergoing the two imaging assessments (Table 1). Ninetyfive of these 205 patients had been included in a previous study (13).

The demographic characteristics of the 205 patients who underwent TTCE and CT stratified by TTCE grade are shown in Table 2. Patients in higher grades were significantly younger than those TTCE negative or in lower grades $(p=0.04)$. Female predominate in grades 2 and 3 and men in grade $4(p=0.003)$. HHT type 2 patients were more frequent in grades 0 and 1 , and type 1 patients predominated in the remaining grades; particularly grade 4 where $90.5 \%$ of the patients were HHT1 $(p<0.0001)$. Hb values were found in case files of 191 patients $(93.2 \%)$ were determined. Seventy-seven patients $(40.3 \%)$ were found to be anemic. No statistically significant differences in $\mathrm{Hb}$ values $(p=0.749)$ were observed when comparing grades, nor were there differences found in the number of patients with anemia $(p=0.91)$ or in the $\mathrm{Hb}$ values of these patients $(p=0.154)$. CT studies of the liver were performed for 188 patients (91.7\%). In $62.2 \%$ of these, the tests revealed HAVMs. In addition to the $\mathrm{Hb}$ values, we do not find significant differences in the number of HAVMs when comparing grades ( $p=0.87)$.

Fifty-nine (38.8\%) of 152 TTCE-positive patients had a PAVM on CT (Table 3): 2 (3\%) grade 1, 18 (42.9\%) grade 2, $17(77.3 \%)$ grade 3, and 21 (100\%) grade 4. One patient categorized as TTCE-negative (no passage of contrast visualized) had a previously embolized PAVM showing no signs of revascularization on CT. Twentyfour patients had a single PAVM and 35 (59.3\%) multiple PAVMs, with no significant differences in the incidence of single or multiple PAVMs between 
grades. However a trend was detected in which the higher the grade, the greater the probability of having multiple PAVMs (test for trend: $P=0.10$ ).

Among 152 TTCE positive patients, the cardiac cycle at which microbubbles first appeared in the left heart chambers after contrast administration was recorded in 137 patients. The mean cardiac cycle at visualization of bubbles in the left ventricle was 6.6 in grade 1, 4.7 in grade 2, 4 in grade 3, and 3.2 in grade 4 (Table 4). In patients classified into higher grades, contrast bubbles appeared earlier $(p<0.0001)$, such that in grade $4,61.9 \%$ of patients showed bubbles in the left atrium during the third cardiac cycle.

In patients without PAVM on CT, bubbles were first seen in the left heart chambers at a mean of 6.1cardiac cycles (SD 1.7) and 3.9 (SD 0.1) in those with PAVM on CT $(p<0.0001)$ (Table 5). A cut-off of $\leq 4$ cycles had a sensitivity of $81.5 \%(95 \% \mathrm{CI}$, $76.1 \%-86.8 \%)$ and specificity of $77.1 \%(95 \% \mathrm{CI}, 71.3 \%-82.8 \%)$ to detect PAVMs and correctly classified $78.8 \%$ of patients. The area under the curve for cardiac cycle in the diagnosis of PAVM was 0.86 (95\% CI: 0.79-0.91) (Fig. 1). Nonetheless, we were unable to establish a clear differentiation between patients with and without CT-proven malformations in each grade based on the cardiac cycle. In grade 2 , the area under the curve for cardiac cycle in the diagnosis of PAVM was 0.63 (95\% CI: $0.46-0.78$ ), with 3 cycles being the cut-off that best classified patients $(60.5 \%)$, with a specificity of $100 \%$ (95\% CI: $83.9 \%-100 \%)$, but much lower sensitivity (11.8\%; 95\% CI: $1.5-36.4 \%)$. A count of 4 cardiac cycles had a sensitivity of $64.7 \%$ (95\% CI 38.3-85.8\%) and specificity of $47.6 \%$ (95\% CI $25.7 \%$ $70.2 \%$ ) to detect PAVM, and correctly classified $55.3 \%$ of patients in this grade. 
Sixteen (27.1\%) of the 59 patients with PAVM had at least 1 embolized PAVM (mean 1.9; range 1-5) when they were first seen in our hospital. Over the period studied, 29/59 (49.2\%) underwent one or various embolizations (mean 3.2; range 1-12), 9 of which were for PAVM revascularization. In 12 patients (2 to treat revascularization) (20.3\%), embolotherapy was recommended based on CT findings (afferent artery $\geq 3 \mathrm{~mm}$ ) (Table 6). When TTCE findings were used as the reference, only $7 / 42$ (16.7\%) patients in grade 2 ( 2 revascularizations) required or were recommended embolotherapy. In Grade 3, treatment was performed or recommended in $14(63.6 \%)$ patients (1 revascularization) and in Grade 4, in 20 (95\%) patients (8 revascularizations). When CT findings were used as the reference, $7 / 18$ (38.9\%) patients with grade 2 PAVM, 14/17 (82.3\%) with grade 3, and $20 / 21(95.2 \%)$ with grade 4 underwent or were recommended treatment. The single grade 4 patient who was not treated had multiple PAVMs on CT, but none were amenable to embolotheraphy.

In the comparative study, 114 patients underwent TTCE using the 2 contrast agents (Table 7). As compared to saline TTCE, Gelofusine TTCE resulted in upgrading of a large percentage of patients in each grade: $64.3 \%$ of TTCE-negative; $54 \%$ in grade $1,60 \%$ in grade 2 , and $64.3 \%$ in grade 3 (videos 1 and 2). A correlation between the two contrast agents was only seen in grade 4 . Because of this considerable overestimation of grade, there was a poor correlation between results for the two contrast agents: 0.32 quantified by kappa statistics (95\% CI 0.25-0.38). In total, $41 / 114$ (36.0\%) undergoing both contrast studies had a PAVM on CT. To detect the same number of PAVMs according to clinical guideline recommendations (positive TTCE, followed by CT), 104 CT studies would be performed based on Gelofusine results and 86 on agitated saline results. No 
significant contrast-related adverse events occurred with either agent. Only one patient, an 11-year-old boy with a huge PAVM in the right lung (TTCE grade 4), experienced dizziness after Gelofusine administration.

\section{Discussion}

Several findings from the present study are consistent with those of our previous research (13) and the results of other authors $(7,10,14,16)$. The data further support the idea that although small PAVMs can be detected on contrast TTCE (grade 1), the afferent artery is not large enough to undergo embolotherapy, and therefore, CT would not be needed $(7,13,14)$. Analysis of the situation regarding TTCE grade 2 requires special consideration. In patients classified as grade 2 , some treatable PAVMs are detected and CT would be indicated in these cases. Nonetheless, the percentage of PAVMs ultimately identified by CT is low: only $25 \%$ to $45.3 \%$ of these patients $(42.9 \%$ in this series) have a PAVM on CT $(10,13,14)$. Furthermore, the percentage amenable to embolotherapy is even lower: $16.7 \%$ in this series and $25.3 \%$ in the study of Velthuis et al (14). Thus, more than half the patients rated as TTCE grade 2 using the current criteria would actually not need CT evaluation. Hence, it would be of value to further refine the criteria to differentiate between TTCE grades 1 and 2 to reduce the number of unnecessary CTs in these patients.

Without a doubt, CT is needed to characterize the malformation in patients in TTCE grades 3 and $4(13,14)$. More than $75 \%$ of these patients $(77.3 \%$ in our series, 77.4\% in the series of Velthuis et al. (14) have PAVM on CT and more than $80 \%$ are accessible to embolotherapy $(82.2 \%$ in grade 3 and $95 \%$ in grade 4 in our study and $83.7 \%$ in the study of Velthuis et al) (14) 
Previous reports found a relationship between TTCE grade and HHT genotype (7, 14) and our results concur with these findings: HHT2 predominated in TTCEnegative patients and grade 1 , whereas HHT1 predominated in the remaining grades. Velthuis et al (14) reported no differences in age or sex between patients categorized into the different grades, but in our series, patients with higher grades were significantly younger than those in the lower grades. These findings support the notion that lung involvement is more frequent and more severe in patients with the HHT1 subtype, as described $(7,31)$. Female predominate in the study group although there was a slight predominance of men in grade 4 . Further studies in larger samples are needed to determine whether these are generalizable differences or result from a collateral effect of the sample. The number of patients with multiple PAVMs (60\%) was similar to previously reported values (32).

Reduced oxygenation of blood due to pulmonary PAVMS, anemia and/or the presence of HAVMs could in HHT patients increased cardiac output as reported (8, 32). An increase in cardiac output has been shown to lead to an increase of blood flow through normally present intrapulmonary arteriovenous anastomosis and therefore be the cause of false positive TTCE results in HHT patients (20). However, in our study, patients testing negative for TTCE had similar levels of $\mathrm{Hb}$ and HAVMs to those with positive TTCE results; suggesting that these parameters do not significantly affect the TTCE findings.

With regard to the cardiac cycle time point, our current results confirm those of our previous study (13): contrast passage occurred earlier in higher grades. Furthermore, and in contrast to the results of Zukotynski et al (10), we found a statistically significant difference in the cardiac cycle at which bubbles were first seen in the left chambers on TTCE between patients with and without PAVMs on 
CT $(\mathrm{p}<0.0001)$. The fourth cycle seemed to be the best cut-off for this purpose, yielding a sensitivity of $81.5 \%$ and specificity of $71.5 \%$, and correctly diagnosing $79 \%$ of patients. However, we were unable to establish a useful cardiac cycle to differentiate between patients with and without CT-confirmed PAVMs within each TTCE grade. Studies in larger patient samples may clarify this aspect.

The treatment of choice for PAVMs is currently embolotherapy, a technique that has minimal functional repercussions and relatively low invasiveness $(34,35)$. Sixteen patients had previously undergone embolotherapy of one or several PAVMs when they were first seen in our unit. Most (15 of 16 patients) tested positive on TTCE; a normal finding according to Lee et al. (36) who reported a high incidence of persistently positive TTCE findings following embolotherapy (90\%). Forty-one patients showed PAVMs on CT with afferent arteries large enough to be treated at follow-up (35); most were in grades 3 and 4 and embolotherapy was feasible in more than $82 \%$. These results concur with those of Velthuis et al (14): $83.7 \%$ of their grade 3 patients had embolotherapy.

To our knowledge, this is the first study comparing contrast TTCE using agitated saline versus Gelofusine for PAVM screening in an HHT population. In all TTCE grades, there was a clear trend to categorize patients into a higher grade with use of Gelofusine than with saline. We attribute this to the smaller size of the Gelofusine bubble, although other characteristics of this contrast medium, such as more stable bubbles and longer bubble retention $(21,24)$, may contribute to this difference. Of particular note, a number of patients classified as TTCE-negative using saline were passed to grade 1 or even 2 with Gelofusine. We cannot say whether this is attributable to a greater capacity of Gelofusine to detect small PAVMs or simply false-positive detection. Small pulmonary shunts on TTCE have 
One limitation of this study is that 2-detector CT was used in some examinations. More sensitive systems would likely detect a larger number of small PAVMS, and this may justify the low incidence of PAVMs in our grade 1 group relative to other studies $(7,14)$. However, we believe that this limitation does not significantly influence the interpretation of the results, as detection of significant PAVMs would not be affected. Second, semiquantitative measurement was used to differentiate between grades 2 and 3, and 3 and 4, although the results were comparable to those found in a study using a quantitative cut-off (14). Third, patients who had undergone previous PAVM embolization were not excluded because in clinical practice, TTCE findings of grade 2 to 4 PAVM in these patients indicate the need for CT assessment of possible revascularization.

In summary, the results of this study further support our previous interpretation that CT is not needed in patients with grade- 1 shunt on TTCE. We believe that the definition of grade 2 should be refined to better differentiate between grades 1 and 2 and further reduce unnecessary CT studies. The cardiac cycle at which bubbles first appear can help to identify patients with and without PAVMs. Use of the fourth cycle correctly classified 79\% of patients. Gelofusine use did not improve 



\section{References.}

1. McDonald J, Wooderchak-Donahue W, VanSant Webb C, Whitehead K, Stevenson DA, Bayrak-Toydemir P. Hereditary hemorrhagic telangiectasia: genetics and molecular diagnostics in a new era. Front Genet. 2015;6:1.

2. Shovlin CL Pulmonary arteriovenous malformations. Am J RespirCrit Care Med 2014;190(11):1217-1228.

3. Circo S, Gossage JR. Pulmonary vascular complications of hereditary haemorrhagic telangiectasia. Curr Opin Pulm Med 2014;20(5):421-428.

4. Cartin-Ceba R, Swanson KL, Krowka MJ. Pulmonary arteriovenous malformations. Chest 2013;144(3):1033-1044

5. Lacombe P, Lacout A, Marcy P, et al. Diagnosis and treatment of pulmonary arteriovenous malformations in hereditary hemorrhagic telangiectasia: an overview. Diagn Interv Imaging 2013;94(9):835-848.

6. Cottin V, Chinet T, Lavole' A, et al. Pulmonary arteriovenous malformations in hereditary hemorrhagic telangiectasia: a series of 126 patients. Medicine (Baltimore) 2007; 86(1):1-17.

7. Van Gent MW, Post MC, Snijder RJ, Westermann CJ, Plokker HW, Mager JJ. Real prevalence of pulmonary right to left shunt according to genotype in patients with hereditary hemorrhagic telangiectasia: a transthoracic echocardiography study. Chest 2010;138(4):833-839.

8. Shovlin CL. Circulatory contributors to the phenotype in hereditary hemorrhagic telangiectasia. Front Genet 2015;6:101.

9. Faughnan ME, Palda VA, Garcia-Tsao G, et al. International guidelines for the 
implications of pulmonary shunting on saline contrast echocardiography. JAm Soc Echocardiogr 2015;28(3):255-263.

12. Velthuis S, Vorselaars VM, van Gent MW, et al. Role of transthoracic contrast echocardiography in the clinical diagnosis of hereditary hemorrhagic telangiectasia. Chest 2013;144(6):1876-1882.

13. Parra JA, Bueno J, Zarauza J, et al. Graded contrast echocardiography in pulmonary arteriovenous malformations. Eur Respir J 2010;35(6):1279-1285.

14. Velthuis S, Buscarini E, Mager JJ, et al. Predicting the size of pulmonary arteriovenous malformations on chest computed tomography: a role for transthoracic contrast echocardiography. Eur Resp J 2014;44(1):150-159

15. Hanneman K, Faughnan ME, Prabhudesai V. Cumulative radiation dose in patients with hereditary hemorrhagic telangiectasia and pulmonary arteriovenous malformations. Can Assoc Radiol J 2014;65(2):135-140.

16. Gazzaniga P, Buscarini E, Leandro G, et al. Contrast echocardiography for pulmonary arteriovenous malformations screening: does any bubble matter? Eur J Echocardiogr 2009;10(4):513-518. 
association. Chest 2013;144(2):542-5488.

19. Lovering AT, Duke JW, Elliot JE. Intrapulmonary arteriovenous anastomoses in humans response to exercise and the environment. J Physiol 2015;593(3):507520.

20. Elliott JE, Duke JW, Hawn JA, Halliwill JR, Lovering AT. Increased cardiac output, not pulmonary artery systolic pressure, increases intrapulmonary shunt in healthy humans breathing room air and 40\% 02. J Physiol 2014;592(Pt20):45374553.

21. Tan HC, Fung KC, Kritharides L. Agitated colloid is superior to saline and equivalent to levovist in enhancing tricuspid regurgitation Doppler envelope and in the opacification of right heart chambers: a quantitative, qualitative, and costeffectiveness study. J Am Soc Echo cardiogr 2002;15(4):309-315.

22.Basset GC, Lin JW, Tran MM, Sistino JJ. Evaluating the potential risks of bubble studies during echocardiography. Perfusion 2015;30(3):219-223

23. La Gerche A, MacIsaac AI, Burns AT, et al. Pulmonary transit of agitated contrast is associated with enhanced pulmonary vascular reserve and right ventricular function during exercise. J Appl Physiol 2010;109(5):1307-1317. 
24. Brugts JJ, Michels $M$, den Uil CA. A cardiac diagnosis by contrast echocardiography. Heart 2014;100(8):657.

25. Gossage J, Kanj G. Pulmonary arteriovenous malformations. A state of the art review. Am J Respir Crit Care Med 1988; 158(2):643-661.

26. Siddiki H, Doherty MG, Fletcher JG et al. Abdominal findings in hereditary hemorrhagic telangiectasia: pictorial essay on 2D and 3D findings with isotropic multiphase CT. Radiographics2008; 28(1):171-183.

27. Ianora AA, Memeo M, Sabba C, Cirulli A, Rotondo A, Angelelli G. Hereditary hemorrhagic telangiectasia: multi-detector row helical CT assessment of hepatic involvement. Radiology 2004;230(1):250-259.

28. Remy-Jardin M, Dumont P, Brillet PY, Dupuis P, Duhamel A, Remy J. Pulmonary arteriovenous malformations treated with embolotherapy: helical CT evaluation of long-term effectiveness after 2-21 year follow up. Radiology 2006;239(2):576585.

29. Woodward CS, Pyeritz RE, Chittams JL, Trerotola SO. Treated pulmonary arteriovenous malformations: patterns of persistence and associated retreatment success. Radiology 2013;269(3):919-926.

30. Shovlin CL, Jackson JE, Bamford KB, et al. Primary determinants of ischaemic stroke/brain abscess risks are independent of severity of pulmonary arteriovenous malformations in hereditary haemorrhagic telangiectasia. Thorax 2008;63(3):259-266. 
31. Bayrak-Toydemir P, McDonald J, Markewitz B, et al. Genotype-phenotype correlation in hereditary hemorrhagic telangiectasia: mutations and manifestations. Am J Med Genet A 2006;140(5):463-470.

32. Cottin V, Blanchet AS, Cordier JF.Pulmonary manifestations of hereditary hemorrhagic telangiectasia. Rev Mal Respir 2006;23(suppl):4S53-4S66.

33. Buscarini E, Leandro G, Conte D, et al. Natural history and outcome of hepatic vascular malformations in a large cohort of patients with hereditary hemorrhagic telangiectasia. Dig Dis Sci. 2011;56(7):2166-2178.

34. White RI Jr, Lynch-Nyhan A, Terry $\mathrm{P}$, et al. Pulmonary arteriovenous malformations: techniques and long terms outcome of embolotherapy. Radiology 1988;169(3):663-669

35. Trerotola SO, Pyeritz RE. PAVM embolization: an update. AJR 2010;195(4):837845.

36. Lee Wl, Graham AF, Pugash RA, et al. Contrast echocardiography remains positive after treatment of pulmonary arteriovenous malformations. Chest 2003;123(2):351-358

37. Woods TD, Harmann L, Purath T, et al. Small and moderate size right to left shunts identified by saline contrast echocardiography are normal and unrelated to migraine headache. Chest 2010;138(2):264-269. 
38. Elliott JE, Nigam SM, Laurie SS, et al. Prevalence of left heart contrast in healthy, young, asymptomatic humans at rest breathing room air. RespirPhysiolNeurobiol2013;188(1):71-78.

39. Dubrey SW, Dahdal G, Grocott-Mason R. Severe anaphylaxis to Gelofusine during a transthoracic echo bubble study. Eur J Echocardiogr2008; 9(2):303. 


\section{Supplemental material Video 1 A, B}

Video 1. A, B. Transthoracic contrast echocardiography, performed in the same patient. Using an agitated saline solution (A) and Gelofusine (B). In this case there is an upgrade from grade 2 with saline to grade 4 with Gelofusine. 


\section{Supplemental material}

Video 2.A, B. Transthoracic contrast echocardiography performed in the same patient: with a saline solution (A) and Gelofusine (B). In this case there is an upgrade from grade 3 with saline to grade 4 with Gelofusine 


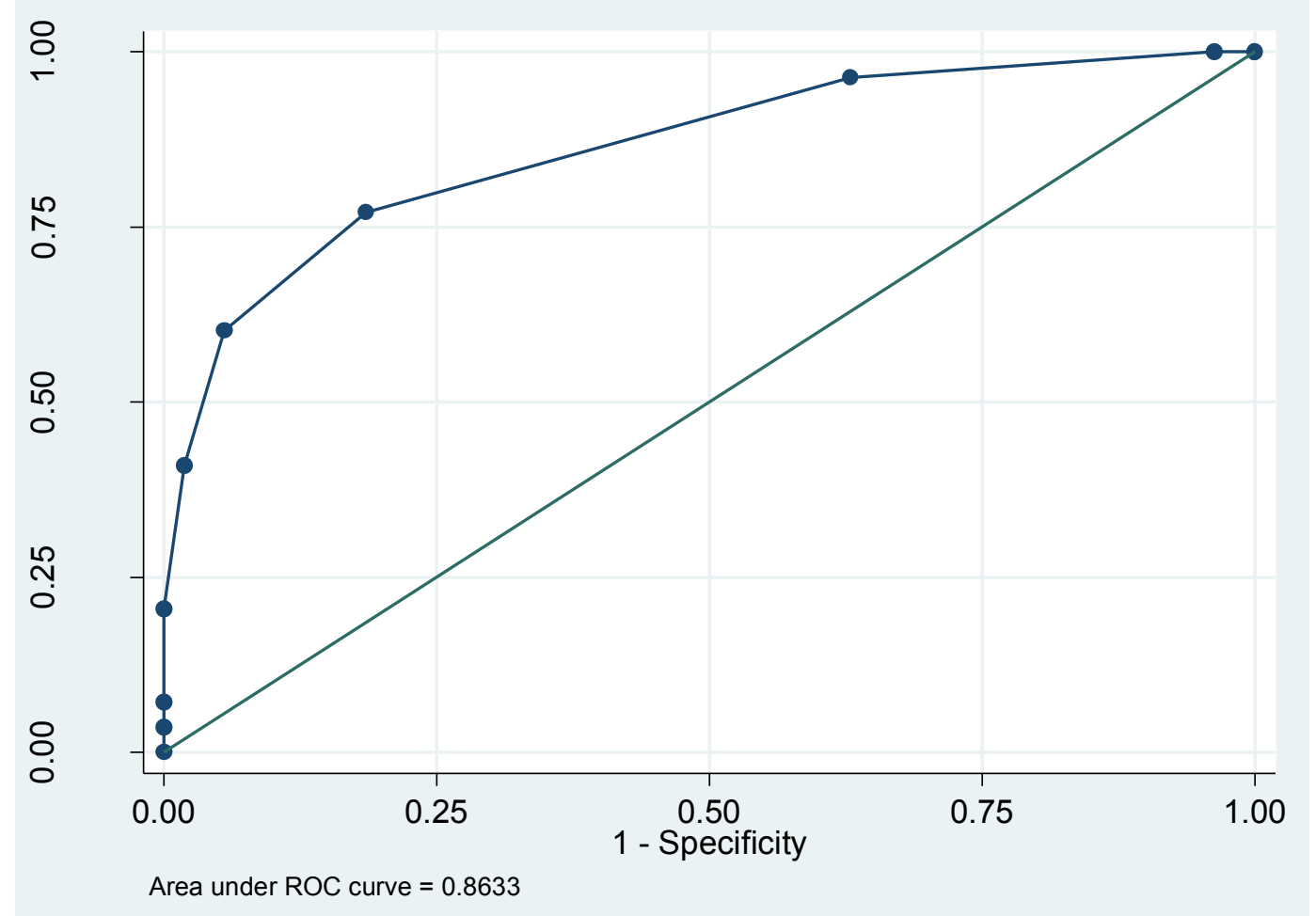

Fig. 1: ROC curve. Cardiac cycle in the diagnosis of pulmonary arteriovenous malformation. 
Table 1. Demographics and genetic characteristics.

\begin{tabular}{|c|c|c|c|}
\hline & HHT patients $n=449$ & TTCE+CT n $=205$ & $P$ value* \\
\hline Age, years, median; range & $43 ; 0-85$ & $50 ; 6-85$ & $<0.001$ \\
\hline Sex & & & 0.74 \\
\hline Male (\%) & $204(45.4)$ & $96(46.8)$ & \\
\hline Female (\%) & $245(54.6)$ & $109(53.2)$ & \\
\hline Genetic testing (\%) & $424(94.4)$ & $197(96.1)$ & \\
\hline HHT1 (\%)/median; range & $192(42.8) / 40 ; 1-85$ & $87(42.4) / 47 ; 6-85$ & $<0.001$ \\
\hline Male & $97(50.5)$ & $44(50.6)$ & 0.993 \\
\hline Female & $95(49.5)$ & $43(49.4)$ & \\
\hline HHT2 (\%)/ median; range & $229(51.00) / 46 ; 0-81$ & $109(53.2) / 50 ; 11-78$ & 0.03 \\
\hline Male & $97(42.4)$ & $51(46.8)$ & 0.443 \\
\hline Female & $132(57.6)$ & $58(53.2)$ & \\
\hline SMAD4 (\%)/ median; range & $3(0.7) / 45 ; 42-48$ & $1(0.5) 42$ & -- \\
\hline Male & $0(0)$ & $0(0)$ & -- \\
\hline Female & $3(100)$ & $1(100)$ & \\
\hline Other** (\%)/ median; range & $25(5.5) / 50 ; 8-74$ & $8(3.9) / 53 ; 35-72$ & 0.93 \\
\hline Male & $10(40)$ & $1(12.5)$ & 0.22 \\
\hline Female & $15(60)$ & $7(87.5)$ & \\
\hline N TTCE performed & 312 & 205 & \\
\hline TTCE positive (\%) & $222(71.1)$ & $152(74.1)$ & 0.49 \\
\hline
\end{tabular}

*The two-tailed chi-square test/Fisher exact test for categorical variables or the Mann-Whitney U test for continuous variables. ${ }^{* *}$ Other: Diagnosis established based on 3 or 4 Curaçao criteria. 
Table 2. Demographics, genetics, hemoglobin values and hepatic arteriovenous malformations, according to TTCE grade

\begin{tabular}{|c|c|c|c|c|c|c|c|c|c|c|}
\hline $\begin{array}{l}\text { TTCE } \\
\text { results }\end{array}$ & Patients, $\mathrm{n}$ & Age median; range & Male (\%)/Female (\%) & HHT1 (\%) & HHT2 (\%) & SMAD4 (\%) & Other* (\%) & $\begin{array}{l}\mathrm{HB} \quad(\mathrm{g} / \mathrm{dL}) ; \\
\text { median; range }\end{array}$ & $\begin{array}{l}\text { Anemia }(\%) / \mathrm{HB}(\mathrm{g} / \mathrm{dL}) \text {, } \\
\text { median; range }\end{array}$ & HAVMs, n (\%) \\
\hline Negative & 53 & $56 ; 19-85$ & $28(52.8) / 25(47.2)$ & $13(24.5)$ & 38 (71.7) & $0(0)$ & $2(3.8)$ & $12.95 ; 6-17.3$ & $\begin{array}{c}22(28.6 \%) / 11.2(6- \\
13.1)\end{array}$ & $32(27.4)$ \\
\hline Grade 1 & 67 & $49 ; 18-78$ & $34(50.7) / 33(49.3)$ & $17(25.4)$ & 46 (68.7) & $0(0)$ & $4(6)$ & $13.05 ; 6.1-17.2$ & $24(31.2) / 10$ (6.1-13.4) & 37 (31.6) \\
\hline Grade 2 & 42 & $46 ; 12-73$ & $17(40.5) / 25(59.5)$ & $27(64.3)$ & $14(33.3)$ & $0(0)$ & $1(2.4)$ & $13.0 ; 5.4-17.4$ & $\begin{array}{c}17(22.1) / 10.7 \text { (5.4- } \\
13.4)\end{array}$ & 25 (21.4) \\
\hline Grade 3 & 22 & $43.5 ; 26-70$ & $4(18.2) / 18(81.8)$ & $11(50)$ & $9(40.9)$ & $1(4.5)$ & $1(4.5)$ & $12.3 ; 8.9-18.0$ & $8(10.4) / 9.5(8.9-11.8)$ & $13(11.1)$ \\
\hline Grade 4 & 21 & $40 ; 6-73$ & $13(61.9) / 8(38.1)$ & $19(90.5)$ & $2(9.5)$ & $0(0)$ & $0(0)$ & $14.0 ; 8.3-19.0$ & $6(7.8) / 9(8.3-10)$ & $10(8.5)$ \\
\hline p-value** & & 0.004 & 0.003 & \multicolumn{4}{|c|}{$<0.0001$} & 0.749 & $0.91 / 0.154$ & 0.87 \\
\hline
\end{tabular}

TTCE: Transthoracic contrast echocardiography; HHT1: deficit of Endoglin; HHT2: deficit of ACLVI/ALK1; Smad4: association of

Hereditary hemorrhagic telangiectasia and Juvenile polyposis. Negative, no signs of contrast passage on TTCE. * Diagnosis established

based on 3 or 4 Curaçao criteria; HB: Hemoglobin; HAVMs: hepatic arteriovenous malformations. ** The two-tailed chi-square test/Fisher exact test for categorical variables or the Kruskal-Wallis test for continuous variables. 
Table 3: Correlation between TTCE and chest CT in the 205 patients undergoing both studies

\begin{tabular}{|l|c|c|c|c|c|}
\hline TTCE results & Patients, $\mathbf{n}(\%)$ & $\begin{array}{l}\text { Patients with PAVMs on } \\
\text { CT n (\%) }\end{array}$ & $\begin{array}{l}\text { Patients with only one PAVM } \\
\text { seen on CT (\%) }\end{array}$ & $\begin{array}{l}\text { Patients with multiple PAVMs } \\
\text { seen on CT (\%) }\end{array}$ & PAVM, mean (SD) \\
\hline Negative & $53(25.9)$ & $1^{*}(1.9)$ & $1(1.9)$ & $0(0.0)$ & 0 \\
\hline Grade 1 & $67(32.7)$ & $2(3)$ & $2(100)$ & $0(0.0)$ & $1(0)$ \\
\hline Grade 2 & $42(20.5)$ & $18(42.9)$ & $7(38.9)$ & $9(52.1)$ & $3.2(2.9)$ \\
\hline Grade 3 & $22(10.7)$ & $17(77.3)$ & $8(47.1)$ & $15(71.4)$ & $2.6(2.0)$ \\
\hline Grade 4 & $21(10.2)$ & $21(100)$ & $6(28.6)$ & $35(59.3)$ & $3.1(3.6)$ \\
\hline All & 205 & $59(28.8)$ & $24(40.7)$ & $3.2(3.0)$ \\
\hline
\end{tabular}

CT, computed tomography; PAVM, pulmonary arteriovenous malformation; TTCE, transthoracic contrast echocardiography. Negative, no signs of contrast passage on TTCE; *previously embolized and no signs of revascularization seen on CT 
Table 4. Correlation between TTCE grade and cardiac cycle.

\begin{tabular}{|l|c|c|}
\hline TTCE grade & Patients n (\%) & Cardiac cycle: \\
& & Mean cardiac cycle, SD; range \\
\hline Grade 1 & $60(43.8)$ & $6.6,1.7 ; 3-10$ \\
\hline Grade 2 & $38(27.7)$ & $4.7,1.1 ; 3-7$ \\
\hline Grade 3 & $18(13.1)$ & $4,0.7 ; 3-5$ \\
\hline Grade 4 & $21(15.4)$ & $3.2,0.6 ; 2-4$ \\
\hline All & $137(100)$ & $5.2,1.8 ; 2-10$ \\
\hline
\end{tabular}

TTCE, transthoracic contrast echocardiography 
Table 5. Sensitivity and specificity of different cardiac cycle cut-offs in the diagnosis of PAVM

\begin{tabular}{|l|c|c|c|c|c|}
\hline Cut-off point* & Sensitivity, \% (95\% CI) & Specificity, \% (95\% CI) & Correctly Classified, \% & LR+ & LR- \\
\hline 1 & $0.0(0-6.6)$ & $100.0(95.7-100)$ & 60.6 & 1.0 & \\
\hline 2 & $3.7(0.5-12.7)$ & $100.0(95.7-100)$ & 62.0 & 1.0 & 0.0 \\
\hline 3 & $37.0(24.3-51.3)$ & $96.4(89.8-99.2)$ & 73.0 & 1.5 & 0.1 \\
\hline 4 & $81.5(68.6-90.7)$ & $77.1(66.6-85.6)$ & 78.8 & 4.1 & 0.3 \\
\hline 5 & $94.4(84.6-98.8)$ & $60.2(48.9-70.8)$ & 73.7 & 10.8 & 0.4 \\
\hline 6 & $98.2(90.1-99.9)$ & $41.0(30.3-52.3)$ & 63.5 & 22.1 & 0.6 \\
\hline 7 & $100.0(93.4-100)$ & $20.5(12.4-30.8)$ & 51.8 & & 0.8 \\
\hline 8 & $100.0(93.4-100)$ & $7.2(2.7-15.1)$ & 43.8 & & 0.9 \\
\hline 9 & $100.0(93.4-100)$ & $3.6(0.8-10.2)$ & 41.6 & & 1.0 \\
\hline 10 & $100.0(93.4-100)$ & $0.0(0.0-4.4)$ & 39.4 & & 1.0 \\
\hline
\end{tabular}

${ }^{*}$ Cardiac cycle at which bubbles were seen in left heart chambers. The cut-off point includes all values less than or equal to this value. LR, likelihood ratio. 
Table 6: Correlation between TTCE grade, CT findings, and embolotherapy treatment

\begin{tabular}{|c|c|c|c|c|c|c|}
\hline $\begin{array}{l}\text { TTCE } \\
\text { results }\end{array}$ & Patients, $\mathrm{n}$ & $\begin{array}{l}\text { Patients with PAVM on } \\
\text { CT, } \mathrm{n}(\%)\end{array}$ & $\begin{array}{l}\text { Patients with } \\
\text { embolized, n (\%) }\end{array}$ & Embolotherapy, n (\%)§ & $\begin{array}{l}\text { PAVMs treated per } \\
\text { patient, mean }\end{array}$ & $\begin{array}{l}\text { Embolotherapy } \\
\text { recommended n (\%) }\end{array}$ \\
\hline Negative & 53 & $1(1.8)$ & $1(1.8)$ & $0(0.0)$ & 0 & $0(0.0)$ \\
\hline Grade 1 & 67 & $2(3)$ & $1(1.5)$ & $0(0.0)$ & & $0(0.0)$ \\
\hline Grade 2 & 42 & $18(42.9)$ & $5(11.9)$ & $6\left(2^{*}\right)(14.3)$ & 2.1 & $1(2.4)$ \\
\hline Grade 3 & 22 & 17 (77.3) & $2(9.1)$ & $9(41.0)$ & 1.8 & $5(1 *)(22.7)$ \\
\hline Grade 4 & 21 & $21(100)$ & $7(33.3)$ & $14\left(7^{*}\right)(66.7)$ & 3.3 & $6\left(1^{*}\right)(28.6)$ \\
\hline
\end{tabular}

I Patients with a PAVM embolized the first time they come to the HHT Unit. § Patients treated with embolotherapy during the study

period. Patients with embolotherapy recommended because of the size of the feeding artery. * Patients with a PAVM and CT signs of revascularization seen by CT. TTCE, transthoracic contrast echocardiography. Negative, no signs of contrast passage on TTCE 
Table 7. Comparison of TTCE Grading Using Agitated Saline Contrast Vs. Gelofusine: first and second injections.

\begin{tabular}{|c|c|c|c|c|c|c|c|c|}
\hline \multirow[t]{2}{*}{ TTCE } & & \multicolumn{6}{|c|}{ Gelofusine } & \multirow[b]{2}{*}{$\begin{array}{l}\text { PAVM on } \\
\text { CT }\end{array}$} \\
\hline & & Negative & Grade 1 & Grade 2 & Grade 3 & Grade 4 & Patients & \\
\hline \multirow{6}{*}{$\begin{array}{l}\text { Agitated } \\
\text { saline }\end{array}$} & Negative & 10 & 17 & 1 & & & 28 & $1(*)$ \\
\hline & Grade 1 & & 14 & 15 & 2 & & 31 & 2 \\
\hline & Grade 2 & & & 12 & 14 & 4 & 30 & 16 \\
\hline & Grade 3 & & & & 5 & 9 & 14 & 11 \\
\hline & Grade 4 & & & & & 11 & 11 & 11 \\
\hline & Patients & 10 & 31 & 28 & 21 & 24 & 114 & \\
\hline & $\begin{array}{c}\text { PAVM on } \\
\text { CT }\end{array}$ & 0 & $1(*)$ & 7 & 11 & 22 & & 41 \\
\hline
\end{tabular}

$\left(^{*}\right)$ One previously embolized PAVM with no CT evidence of revascularization.

TTCE, transthoracic contrast echocardiography; negative, no signs of contrast passage on TTCE 
Video $1 \mathrm{~A}$
Click here to download Video Still: Video 1 A.avi Click here to download Video Still: Video 1 A.avi

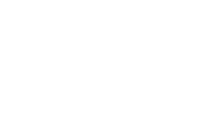

$\sqrt{2}$

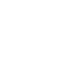
(1) (1) (1) (1) (1) (1) $\sqrt{3}$

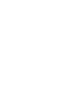

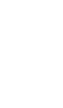

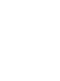

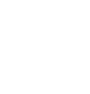

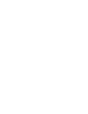

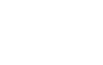

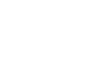
. -

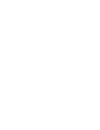

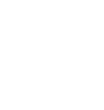
. .

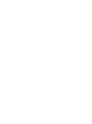

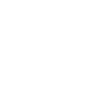

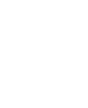
. . .

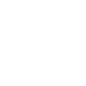
.

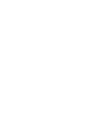

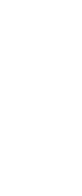
.

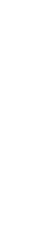

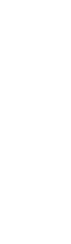


Click here to download Video Still: Video 1 B.avi

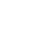

vi

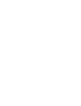

列

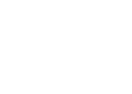

$\sqrt{10}$

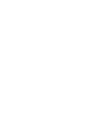

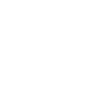
(2) - 
Video 2 A
Click here to download Video Still: Video 2 A.avi

Click here to download Video Still: Video 2 A.avi

$\left(\frac{10}{20}\right.$

$\sqrt{2}$

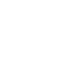
(1) (1) (1) (1) (1) (1) $\sqrt{3}$ (1)

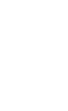
(1)

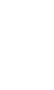
(1) . (1) (n) (1)

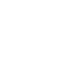
. . (n) (1) (1) (1)

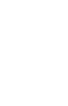
.

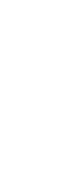
.

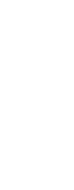
.

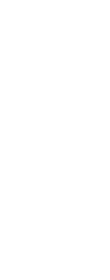


Click here to download Video Still: Video 2 B.avi Click here to download Video Still: Video 2 Bavi

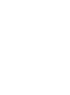

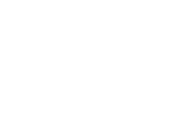

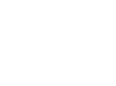
$\left(\frac{10}{2}\right.$ (1) (1)

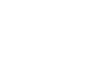
(1) (1) $\sqrt{2}$ (1) (1)

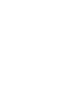

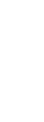

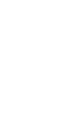

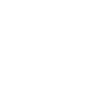

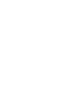

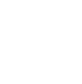

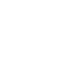

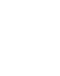

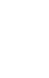

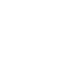

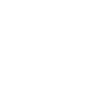

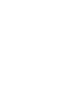

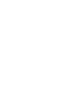
.

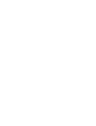

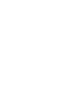



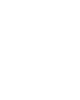

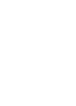

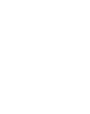

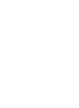

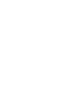
.

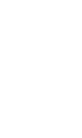

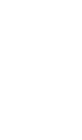

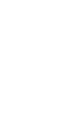

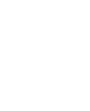
. . - 